\title{
Adaptive Line Enhancement for Improved Uplink Time Difference of Arrival Localization
}

\author{
Paria Shahabi (Corresponding Author) \\ Multimedia University \\ 63100 Cyberjaya, Selangor, Malaysia
}

Tel: 60-14-638-9332 E-mail: paria_shsh@hotmail.com

\author{
Mardeni Roslee \\ Multimedia University \\ 63100 Cyberjaya, Selangor, Malaysia
}

Tel: 60-383125481Ｅ-mail: mardeni.roslee@mmu.edu.my

\author{
Mohsen Riahi Manesh \\ Multimedia University \\ 63100 Cyberjaya, Selangor, Malaysia \\ Tel: 60-17-657-0406_E-mail: mohsen.rm64@hotmail.com
}

\author{
Teong Chee Chuah \\ Multimedia University \\ 63100 Cyberjaya, Selangor, Malaysia \\ Tel: 60-383125352_Email: tcchuah@mmu.edu.com
}

Received: June 12, 2011

Accepted: July 12, 2011

doi:10.5539/mas.v5n5p204

\begin{abstract}
The problem of locating mobile stations (MS) in cellular systems based on uplink time difference of arrival (UTDOA) of MS signal at spatially separated base stations with known locations is addressed in this paper. Multipath fading and channel noise are the main factors resulting in inaccurate mobile station position estimation. Therefore, use of the normalized least mean square (NLMS) algorithm based adaptive line enhancer (ALE) followed by a correlator is proposed to obtain more precise time difference of arrival (TDOA) estimation. The proposed technique applies the ALE as a pre-filter to signal cross correlation, leading to improved accuracy in TDOA estimation and consequently more precise positioning of MS. The robustness of the proposed technique is examined and analyzed through computer simulations. Simulation results indicate superior performance of the proposed ALE-UTDOA estimator over the conventional cross correlation method.
\end{abstract}

Keywords: ALE, Position location, UTDOA

\section{Introduction}

Recently, mobile station (MS) positioning has become a significant part of mobile communication technologies. MS positioning offers a wide range applications ranging from location based services (LBS) such as location based billing, intelligent transportation and fleet management on wireless networks, to emergency services (Caffery \& Stuber, 1998). MS positioning techniques include those based on received signal strength (RSS), 
angle of arrival (AOA), time of arrival (TOA) and time difference of arrival (TDOA) of the received signals. The RSS-based positioning method is the simplest but the most inaccurate technique while the AOA, TOA and TDOA methods obtain more accurate estimation. However, due to the use of directional antennas, the AOA method is more expensive and complicated; whereas the TOA technique requires mutual synchronization between the MS and the base station (BS). Moreover, two-way TOA ranging incurs long propagation delays (Sahinoglu et al., 2008). The uplink time difference of arrival (UTDOA) method, also known as hyperbolic positioning, does not suffer these drawbacks; the only requirement it needs to fulfill is the synchronization between BSs. Implementation of the UTDOA method has been made simple through the accomplishment of synchronization between BSs in modern cellular networks.

In the UTDOA estimation technique, localization is obtained through detection of time differences of arrival of MS signals at pairs of BSs with respect to the home base station, which greatly reduces the time synchronization requirements between MSs and BSs. In this method, a hyperbola is formed with the focus of two BSs and the focal length of the distance difference between the MS to two BSs. Thus, through the intersection of at least two hyperbolas (which requires at least three BSs), the two dimensional position of the MS can be achieved. Therefore, conventional UTDOA positioning is composed of two steps (Rappaport et al., 1996): first, obtaining TDOA parameters of a signal from the MS between pairs of BSs by a time delay estimation algorithm and forming a set of nonlinear hyperbolic equations, second, using an appropriate algorithm to solve the hyperbolic equations obtained from the first step to uniquely localize the MS. Several algorithms for solving the hyperbolic equation can be found in (Sayed et al., 2005; Schau \& Robinson, 1987).

In order to compute the TDOAs in the first step, a simple method suggested and examined in the literature for multipath channels is the cross correlation (CC) approach. The cross correlator is easy to implement but it has the disadvantage of large biases especially in dense multipath environments. As a result, the cross correlation performance and consequently the TDOA estimation accuracy deteriorate in low signal-to-noise ratio (SNR) circumstances. Some authors proposed pre-filtering the signals before cross correlation to improve the SNR (Azaria \& Hertz, 1984). Spectral subtracting techniques have been proven to be well suited to time delay estimation (He et al., 2004). Nevertheless, the adaptive line enhancement (ALE) method introduced by Widrow in 1975 has been shown to have superior performance in time delay estimation than the conventional pre-processors for CC (Krolik et al., 1985; Tiengwattanatum et al., 2008). In this work, utilization of the ALE as a pre-filter for cross correlation is proposed and examined in UTDOA based localization to improve the accuracy of TDOA estimation, and subsequently reducing the uncertainty in MS positioning. The robustness of the proposed technique is assessed through computer simulation results.

\subsection{Cross Correlation}

Having sent a signal $s[n]$ from a source, the general form of received signals at two spatially separated receivers, $r_{1}[n]$ and $r_{2}[n]$, can be modeled as follows

$r_{1}[n]=A_{1} s\left[n-\tau_{1}\right]+n_{1}[n]$

$r_{2}[n]=A_{2} s\left[n-\tau_{2}\right]+n_{2}[n]$

where $A_{1}, A_{2}$ are amplitude scalings, $\tau_{1}, \tau_{2}$ are times of arrival and $n_{1}[n], n_{2}[n]$ are due to noise and multipath components. Considering the receiver with the shortest time of arrival the equations can be rewritten in the form of

$r_{1}[n]=s[n]+n_{1}[n]$

$r_{2}[n]=A s\left[n-T_{d}\right]+n_{2}[n]$

where $A$ is the amplitude ratio and $T_{d}=\tau_{2}-\tau_{1}$ is time difference of arrival (TDOA) of signal $s[n]$ between two receivers which needs to be estimated. The general equation for computing the cross correlation is in the form of

$R_{r_{1} r_{2}}[l]=\sum_{n=-\infty}^{+\infty} r_{1}[n] r_{2}[n-l]$

The value of argument $l$ for which Eqn. (5) is maximized is an estimation of the TDOA $T_{d}$. A typical block diagram of the cross correlator is shown in Figure 1.

\section{Adaptive Line Enhancement}

The ALE can be used in cases where the objective is to eliminate the imperfection $n[n]$, from a narrowband signal corrupted by noise and multipath components $r[n]$, containing sinusoidal components, $s[n]$. Figure 2 demonstrates the block diagram of the ALE under consideration. It can be seen that the ALE uses the received signal as desired reference signal and a delayed copy of it as the input of its predictor. The task of the delay stage 
is to de-correlate noise samples between the reference and input signals. Consequently, the output of the predictor forms the predicted sinusoidal components and when the filter coefficients are adapted in a way to minimize the error signal $e[n]$ in some sense, the ALE will be an optimum filter to render the sinusoidal components.

The ALE is a time varying system in which the weights are updated using the normalized least mean square (NLMS) algorithm. Unlike the LMS algorithm which is sensitive to the scaling of its input and hence is vulnerable to stability issues, the NLMS algorithm addresses this issue by normalizing the input signal with the input power (Haykin, 2002). The delay unit $\Delta$ must be chosen to ensure de-correlation among noise samples in the reference input and predictor input. The sinusoidal components remain correlated due to their periodic behavior. The output from the ALE will be

$y[n]=y_{n}=\mathbf{w}_{n} \mathbf{r}_{n-\Delta}$

where

$\mathbf{w}_{n}=\left[\begin{array}{llll}w_{0} & w_{1} & \ldots & w_{M}\end{array}\right]$

$\mathbf{r}_{n}=\left[\begin{array}{llll}r_{n} & r_{n-1} & \ldots & r_{n-M}\end{array}\right]^{T}$

are the $(M+1)$-length filter coefficients and input corrupted signal vector, respectively. The error signal is defined as

$e_{n}=r_{n}-y_{n}$

Equation (10) depicts recursive update of the coefficient vector based on the NLMS algorithm.

$\mathbf{w}_{n+1}=\mathbf{w}_{n}+\mu e_{n} \frac{\mathbf{r}_{n-\Delta}^{*}}{\left\|\mathbf{r}_{n}^{2}\right\|}$

where $\mu$ is the step size and $\left\|\mathbf{r}_{n}^{2}\right\|$ denotes the input signal power for normalization.

\section{Proposed Method}

Conventionally, in the UTDOA based mobile station positioning, the TDOAs are obtained via cross correlation of pairs of received signals from different BSs with respect to the home BS in which the MS exists. The accuracy of localization depends on the accuracy of the estimated TDOAs obtained from the cross correlation. As mentioned earlier, the performance of cross correlation degrades in low SNR conditions. Therefore, the received signal at each BS is pre-filtered by the ALE block to enhance the performance of the cross correlator and consequently improve the accuracy of the estimated TDOAs. The estimated TDOAs are then processed by an appropriate algorithm to calculate a unique solution for the hyperbolic equations. Figure 3 demonstrates a typical block diagram of the proposed ALE-UTDOA localization method. It is seen that the ALE at each BS is applied to filter noise and multipath components out from the received signal and hence increase the SNR. Having improved the quality of the received MS signal at each BS, cross correlation must be conducted over pairs of received signals to calculate the TDOAs. Assuming base station 1 (BS\#1) being the home BS, it is seen from Figure 3 that the first TDOA (TDOA\#1) is computed by applying cross correlation over signals filtered at BS\#2 and BS\#1. As such, TDOA\#(N-1) are calculated by performing cross correlation over the signals filtered at $\mathrm{BS} \# N$ and BS\#1 where $N$ denotes the number of BSs participating in MS localization. Based on the obtained TDOAs, the hyperbolic equations are formed and solved accordingly to estimate the MS position.

\section{Simulation and Results}

In this paper, the proposed ALE-UTDOA method as well as the conventional UTDOA method have been implemented in MATLAB and comprehensively compared. The signal sent from the MS is assumed to be a one-millisecond sinusoidal pulse in the form of

$s[n]=A_{0} \sin \left[2 \pi f_{s} n+\theta_{0}\right]$

where $A_{0}$ is the amplitude, $f_{s}$ is the signal frequency and $\theta_{0}$ is the phase of the signal. For simplicity, we can assume that the amplitude and phase are constants and equal to 1 and 0 , respectively.

The received signal $r[n]$ at $k$-th BS can be expressed as (Comsa et al., 2007)

$r_{k}[n]=\sum_{j=1}^{P_{N}} A_{j k} \mathrm{~s}\left[n-\tau_{k}\right]+n_{k}[n]$

where $A_{j k}$ is the channel induced amplitude, $P_{N}$ is the number of paths between the MS and the $k$-th BS, $\tau_{k}$ is the time of arrival of the MS signal at BS $k$ and $n_{k}[n]$ is additive white Gaussian noise (AWGN). The time delay $\tau_{k}$ can be calculated as follows 
$\tau_{k}=\frac{\sqrt{\left(y_{k}-y_{M S}\right)^{2}+\left(x_{k}-x_{M S}\right)^{2}}}{c}$

where $c$ is the speed of light and $\left(x_{M S}, y_{M S}\right)$ and $\left(x_{k}, y_{k}\right)$ are the coordinates of the MS and the $k$-th BS, respectively.

The channel between the MS and each BS is assumed to obey the model in Eqn. (12). Particularly, it follows the COST-207 model (i.e. multipath Rayleigh fading with $P_{N}$ paths). A cluster size of seven and the cell radius of $R$ $=5000$ meters are considered. The coordinates of the BSs are assumed in the following form:

$$
(0,0),\left(\frac{3}{2} R, \frac{\sqrt{3}}{2} R\right),\left(\frac{3}{2} R,-\frac{\sqrt{3}}{2} R\right),(0,-\sqrt{3} R),\left(-\frac{3}{2} R,-\frac{\sqrt{3}}{2} R\right),\left(-\frac{3}{2} R, \frac{\sqrt{3}}{2} R\right),(0, \sqrt{3} R)
$$

In all scenarios, the MS coordinates is set to be on $(4000,2000)$ meter and the home BS is assumed to be at the origin of the coordinate, i.e., $(0,0)$. The performance of positioning is measured quantitatively using the root mean square error (RMSE) of the mobile location.

The contour map representing the actual and estimated MS coordinates for the proposed and conventional UTDOA positioning methods are illustrated in Figure 4. The SNR is fixed to $15 \mathrm{~dB}$. It can be noticed that by increasing the filter taps, the accuracy of mobile positioning with respect to the conventional UTDOA method is greatly improved. For instance, while the UTDOA method localizes the MS position coordinates at (4156.20, 1795.00) with a radius equal to RMSE $=257.72 \mathrm{~m}$ at the vicinity of the actual MS location, the ALE-UTDOA technique with 100 taps estimates the MS at $(3995.20,1962.00)$ with a radius equal to RMSE $=38.32 \mathrm{~m}$. It is obvious that the reconnaissance area to seek the MS is significantly reduced.

Figure 5 compares the cumulative probability of RMSEs of less than $100 \mathrm{~m}$ under different SNR values for both the conventional UTDOA and the proposed ALE-UTDOA methods. When comparing with the Federal Communication Commission (FCC)'s requirements that all wireless service providers must report the MS location to an E-911 at public safety answering point with a minimum accuracy of $100 \mathrm{~m}$ for $67 \%$ of all calls, it can be seen that utilizing the ALE technique in UTDOA localization provides an approximate SNR reduction of $25 \mathrm{~dB}$ with respect to the case of the UTDOA without the ALE.

In Figure 6, cumulative probability of different RMSEs of both the proposed and conventional method for a fixed SNR of $15 \mathrm{~dB}$ is demonstrated. It can be seen that positioning RMSE is drastically reduced by applying the ALE technique in UTDOA localization. For example, for RMSEs of less than $100 \mathrm{~m}$, the confidence level of the proposed method is approximately 75\% (which fulfills FCC's requirement for E-911 services) while the same condition can only be achieved in $12 \%$ of times using conventional UTDOA technique.

The impact of the number of BSs on the accuracy of mobile positioning is shown in Figure 7. For both methods, the more number of BSs utilized in positioning, the higher is the accuracy of MS estimation. Nevertheless, Table 1 demonstrates that, when compared to the conventional method, use of the proposed ALE-UTDOA method leads to an improvement from $76.4 \%$ to $84 \%$ by using 4 to 7 BSs, respectively.

Figure 8 examines the effect of the number of paths in the multipath channel on the accuracy of localization for both methods. As described earlier, based on Eqn. (12), increasing the number of signal paths to reach to each BS results in heavy degradation of the received signal quality. As a result, for both methods, the range error between the estimated MS and the actual MS position is increased. However, the RMSE deterioration in the conventional method is from $300 \mathrm{~m}$ to $700 \mathrm{~m}$, whereas that of the proposed ALE-UTDOA is from $100 \mathrm{~m}$ to $200 \mathrm{~m}$. Consequently, is can be concluded that the ALE technique compensates the signal deteriorations caused by noise and multipath due to the extra paths.

\section{Conclusion}

A major challenge encountering UTDOA positioning method in mobile wireless systems is the noise and multipath effect due to non-line-of-sight (NLOS) channels and consequently, techniques to mitigate such channel effects are crucial to the overall performance of localization. In this paper, an improved UTDOA localization scheme using ALE to pre-process signals heavily affected by noise and multipath before using a TDOA estimator has been proposed. The validity and robustness of the proposed method have been confirmed by simulation results. The improved accuracy outweighs the additional complexity imposed to the system. Future work can be undertaken to reduce the uncertainty of mobile positioning and the complexity of the ALE via variable step sizes and filter taps.

\section{References}

Azaria, M. \& Hertz, D. (1984). Time Delay Estimation by Generalized Cross Correlation Method. IEEE Trans. 
On Acoustics, Speech, and Signal Processing. 32, 2, 208-285. DOI: 10.1109/TASSP.1984.1164314

Caffery, J. J. \& Stuber, G. L. (1998). Overview of radiolocation in CDMA Cellular systems. IEEE Communication Mag., 36, 4, 38-45. DOI: 10.1109/35.667411, http://dx.doi.org/10.1109/35.667411

Comsa, C., Luo, J., Haimovich, A. \& Schwartz, S. (2007). Wireless Localization using Time Difference of Arrival in Narrow-Band Multipath Systems. Proc. ISSCS, 2, 1-4. DOI: 10.1109/ISSCS.2007.4292764

Haykin, S. (2002) Adaptive Filter Theory. Prentice Hall, ISBN 0-13-048434-2.

He, X., Gamba, J. \& Shimamura, T. (2004). Explicit Frequency Domain Noise Compensation for Time Delay Estimation. WSEAS Trans. On Circuits and Systems, 3, 1, 85-93.

Krolik, J., Eizenman, M. \& Pasupathy, S. (1985). Application of the LMS Adaptive Line Enhancer in Time Delay Estimation. Proc. ICASSP, 10, 1766-1769. DOI: 10.1109/ICASSP.1985.1168177

Rappaport, T. S., Reed, J. H. \& Woerner, B. D. (1996). Position Location Using Wireless Communications on Highways of the Future. IEEE Communications Mag., 34, 10, 33-41. DOI: 10.1109/35.544321, http://dx.doi.org/10.1109/35.544321

Sahinoglu, Z., Gezici, S. \& Guvenc, I. (2008). Ultra-wideband Positioning Systems. Cambridge University Press. DOI: 10.1017/CBO9780511541056, http://dx.doi.org/10.1017/CBO9780511541056

Sayed, A. H., Tarighat, A. \& Khajehnouri, N. (2005). Network-Based Wireless Location: Challenges faced in developing techniques for accurate wireless location information. IEEE Signal Processing Mag., 24-40. DOI: 10.1109/MSP.2005.1458275, http://dx.doi.org/10.1109/MSP.2005.1458275

Schau, H. \& Robinson, A. (1987). Passive Source Localization Employing Interesting Spherical Surface from Time-of-Arrival Differences. IEEE Trans. On Acoustics, Speech, and Signal processing, 35, 8, 1223-1225. DOI: 10.1109/TASSP.1987.1165266, http://dx.doi.org/10.1109/TASSP.1987.1165266

Tiengwattanatum, C., Naoyuki, N. \& Shimamura, T. (2008). Adaptive Line Enhancer for Time Delay Estimation of Ultrasonic Echoes. Proc. ISCIT, 368-371. DOI: 10.1109/ISCIT.2008.4700215

Widrow, B. et al. (1975). Adaptive Noise Canceling: Principles and Application. Proc. IEEE, 63, 1692-1716. DOI: 10.1109/PROC.1975.10036, http://dx.doi.org/10.1109/PROC.1975.10036

Table 1. RMSE and percentage of improvement for both the UTDOA and ALE-UTDOA methods for different number of base stations

\begin{tabular}{|c||c|c|c|}
\hline $\begin{array}{c}\text { The number of base } \\
\text { stations }\end{array}$ & $\begin{array}{c}\text { UTDOA method } \\
\text { RMSE }\end{array}$ & $\begin{array}{c}\text { ALE-UTDOA } \\
\text { method } \\
\text { RMSE }\end{array}$ & $\begin{array}{c}\text { Percentage of } \\
\text { improvement }\end{array}$ \\
\hline \hline $\mathbf{4}$ & 502.3224 & 118.4561 & $76.4 \%$ \\
\hline $\mathbf{5}$ & 314.5377 & 70.0829 & $77.7 \%$ \\
\hline $\mathbf{6}$ & 281.6282 & 57.3966 & $79.6 \%$ \\
\hline $\mathbf{7}$ & 254.2942 & 38.8840 & $84.7 \%$ \\
\hline
\end{tabular}




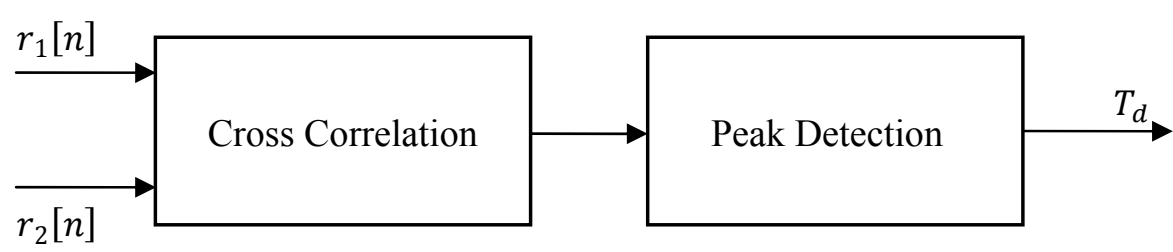

Figure 1. Block diagram of a cross correlator

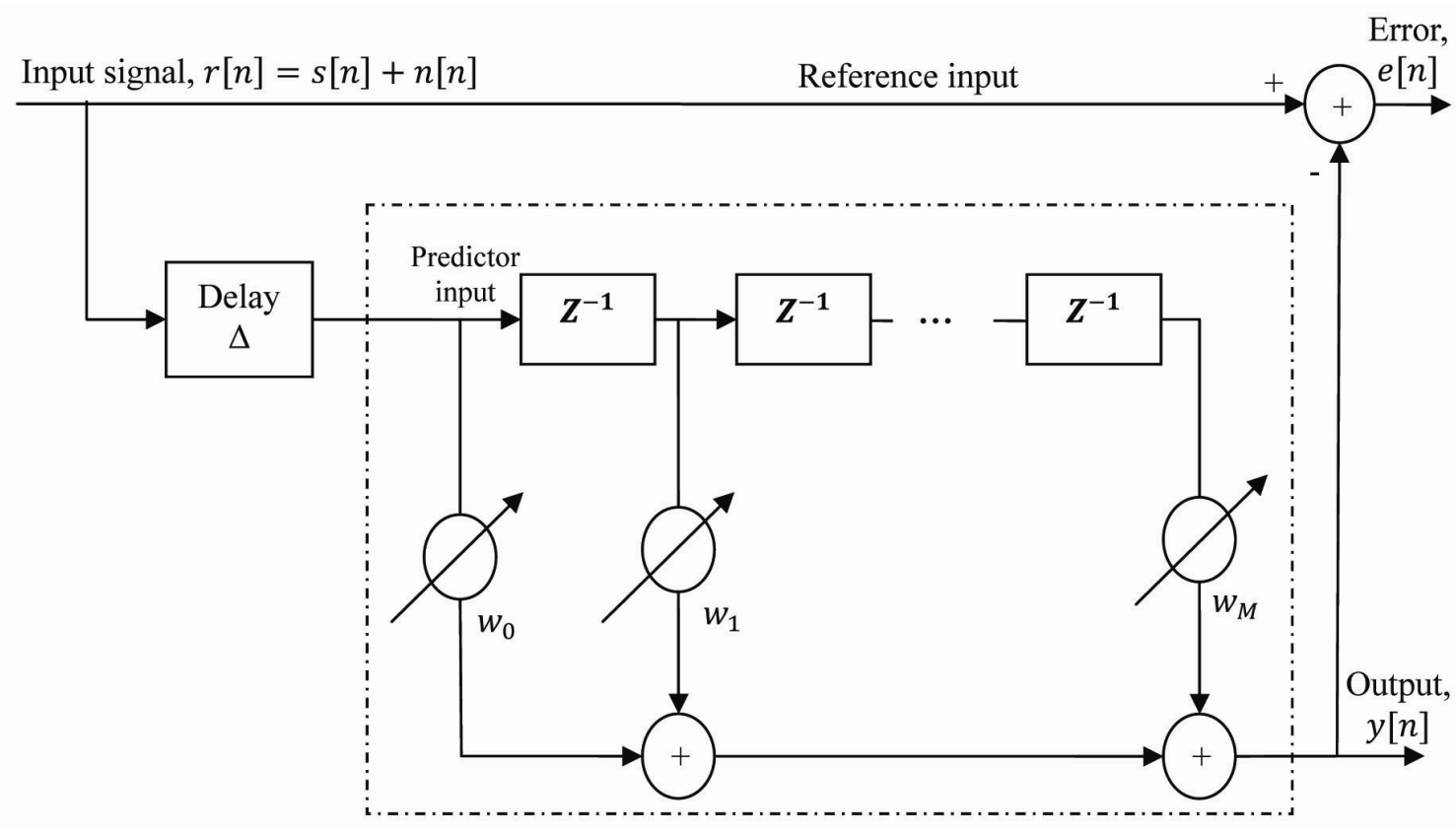

Figure 2. Block diagram of the adaptive line enhancer 


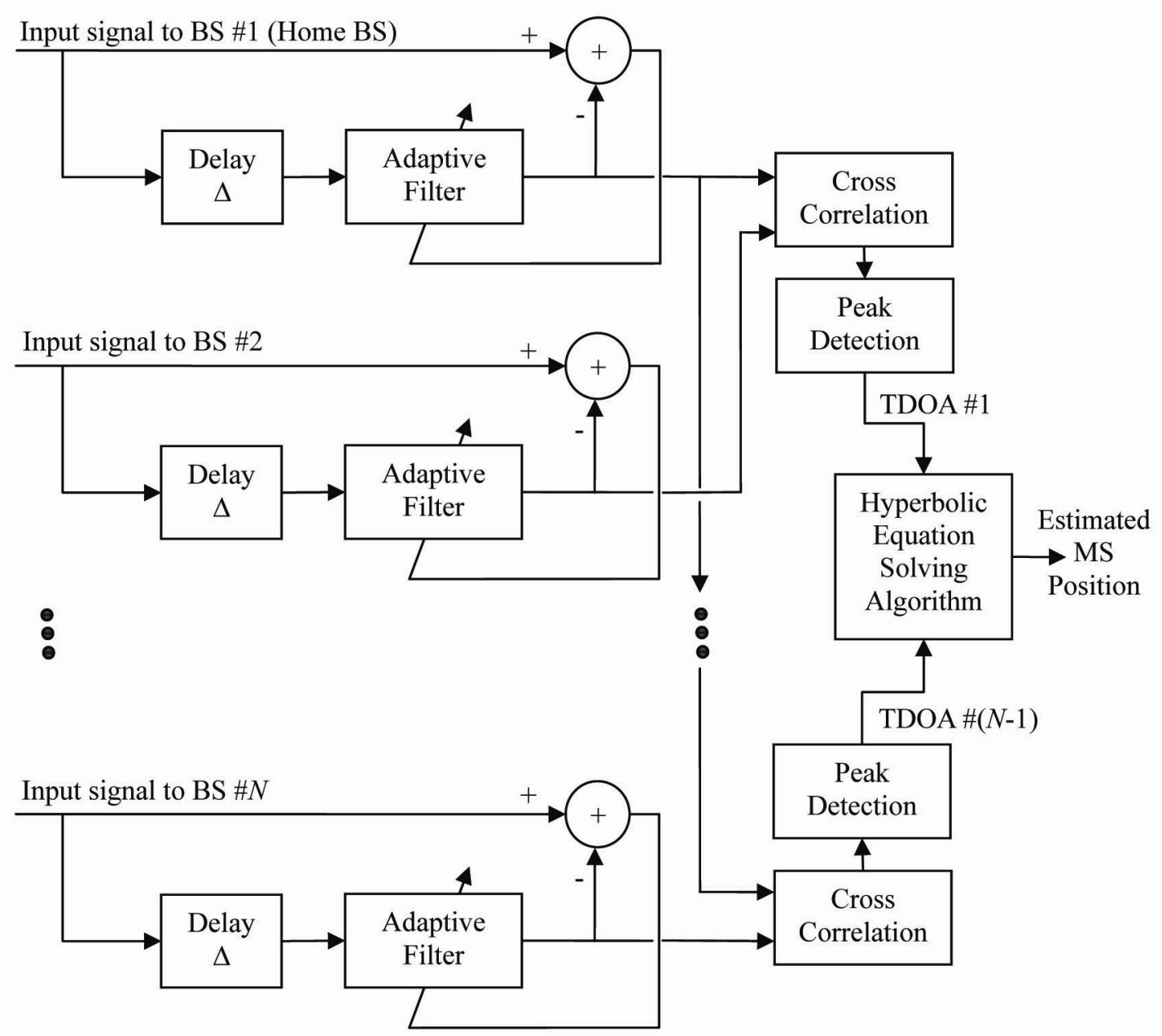

Figure 3. Block diagram of the proposed ALE-UTDOA localization method 


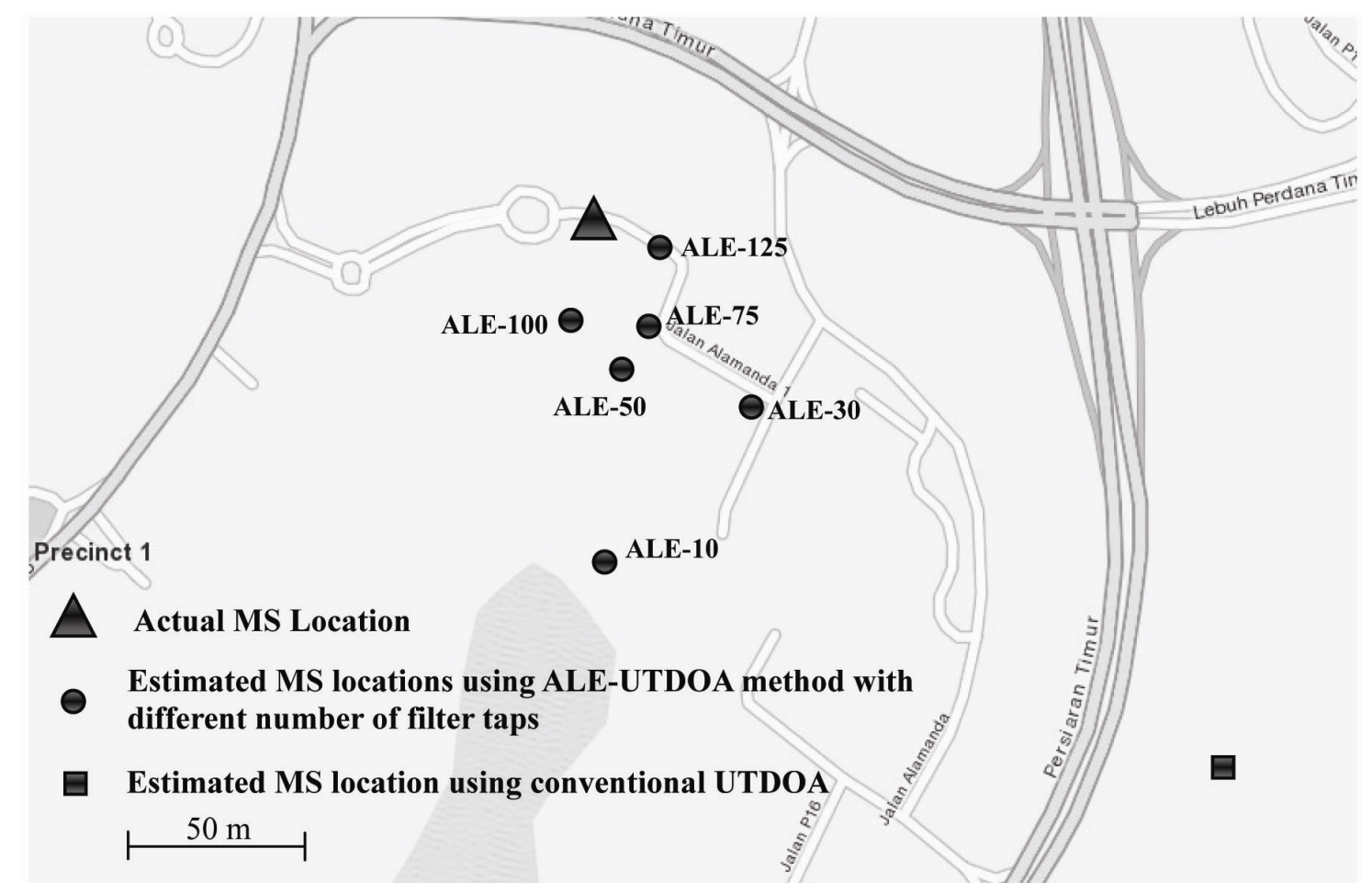

Figure 4. Actual and estimated mobile station positions for both the UTDOA and ALE-UTDOA methods

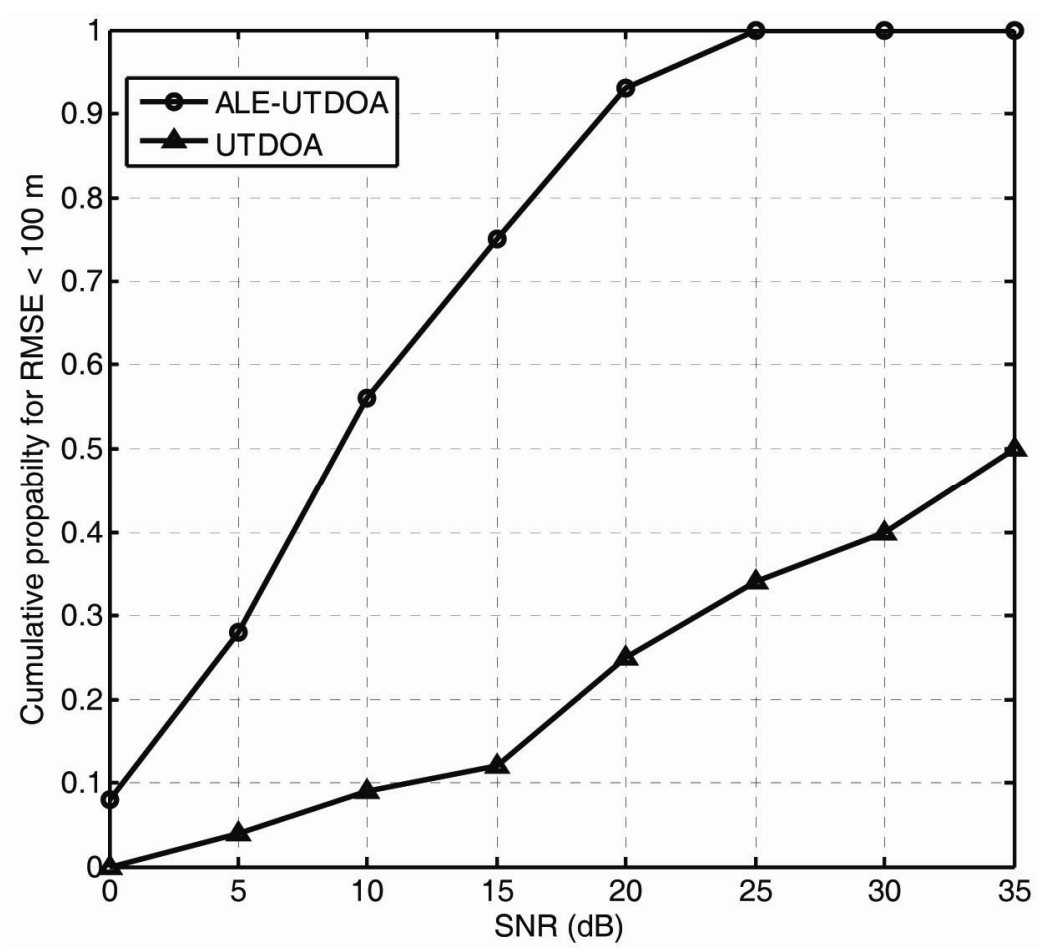

Figure 5. Cumulative probability of RMSEs of less than $100 \mathrm{~m}$ at different SNR values 


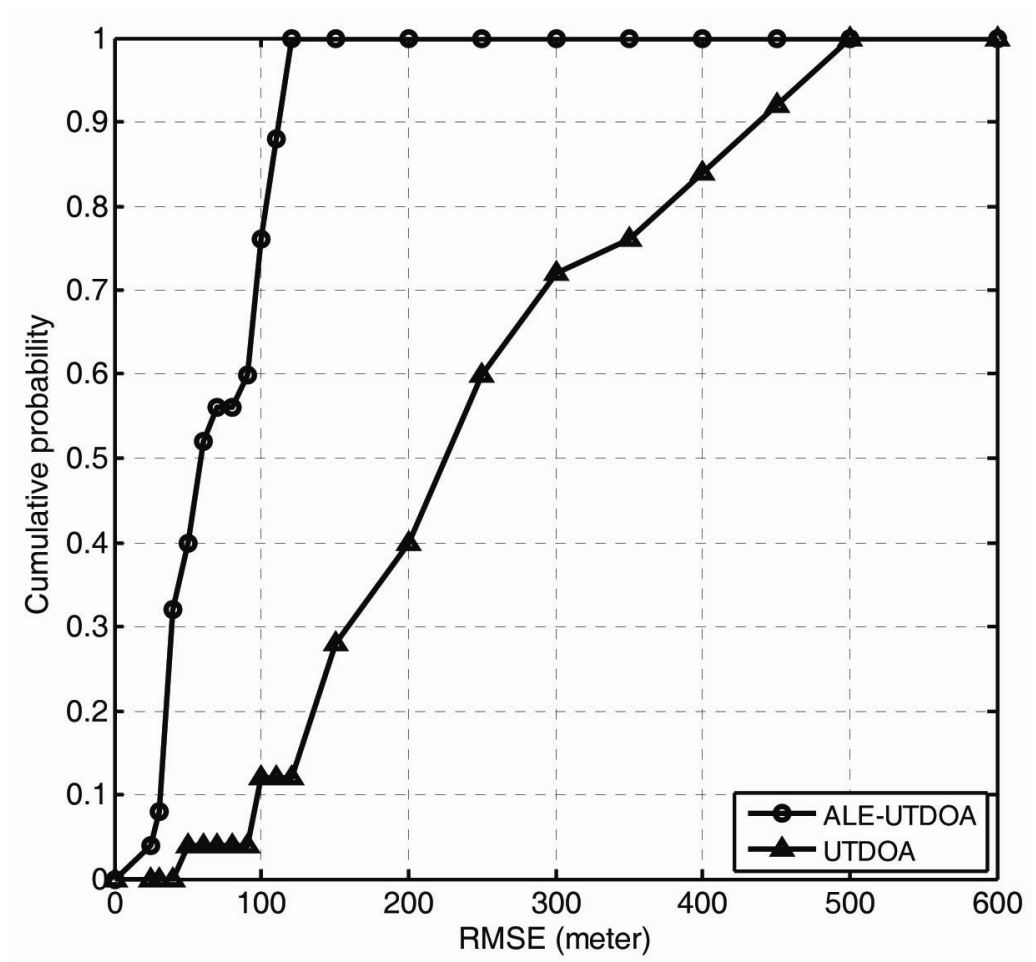

Figure 6. Cumulative probability of different RMSEs at $\mathrm{SNR}=15 \mathrm{~dB}$

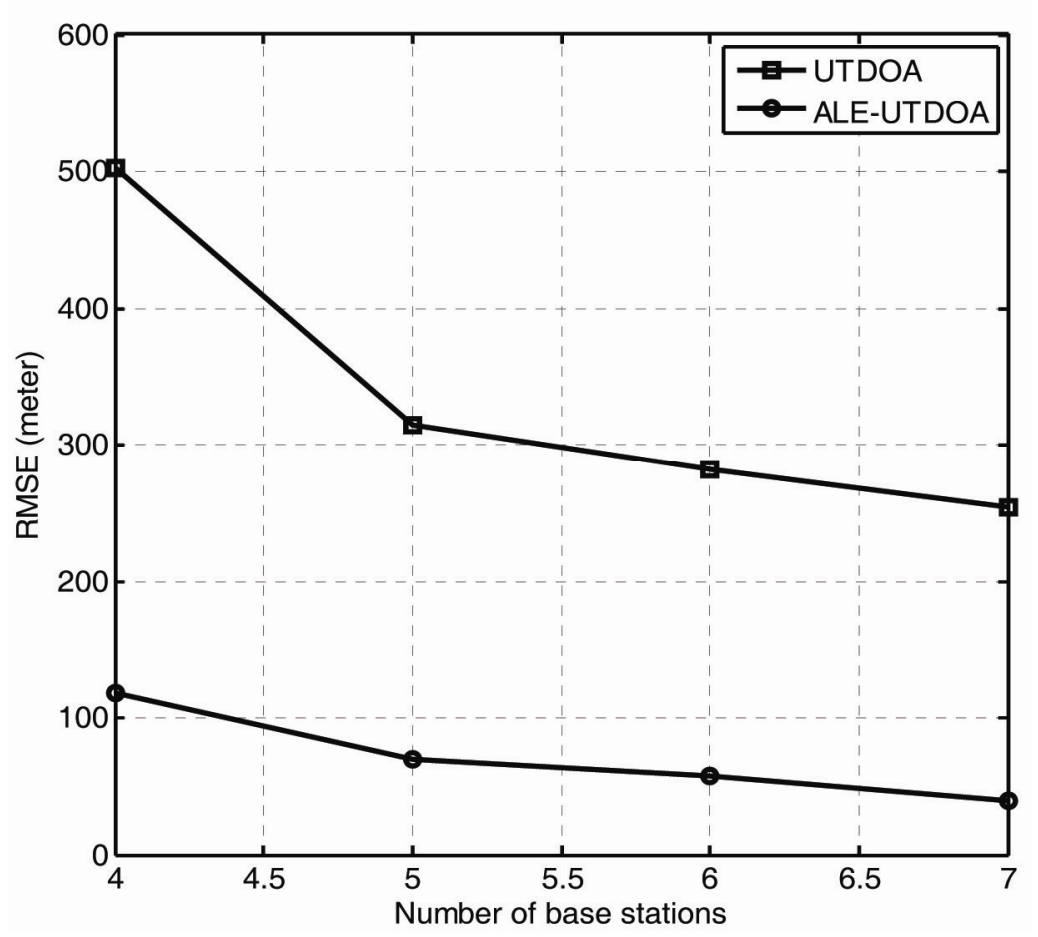

Figure 7. RMSE vs. number of base stations for both the UTDOA and ALE-UTDOA methods 


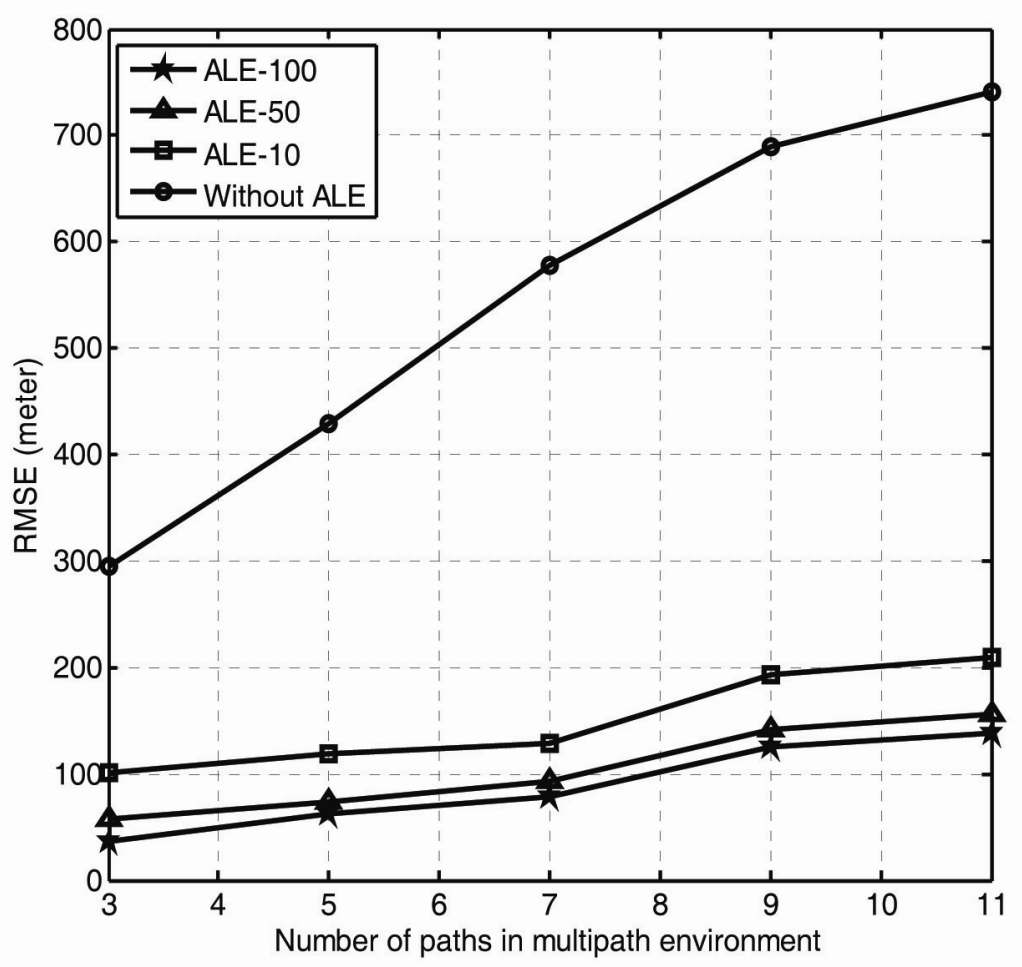

Figure 8. Effect of number of paths and ALE filter taps on the RMSE 